\begin{tabular}{|c|c|}
\hline Title & Numerical verification of degeneracy in hexagonal photonic crystal fibers \\
\hline Author(s) & Koshiba, Masanori; Saitoh, Kunimasa \\
\hline Citation & $\begin{array}{l}\text { IEEE PHOTONICS TECHNOLOGY LETTERS, 13(12), 1313-1315 } \\
\text { https://doi.org/10.1109/68.969892 }\end{array}$ \\
\hline Issue Date & 2001-12 \\
\hline Doc URL & http:/hdl.handle.net/2115/5587 \\
\hline Rights & $\begin{array}{l}\text { (0)2001 IEEE. Personal use of this material is permitted. However, permission to reprint/republish this material for } \\
\text { advertising or promotional purposes or for creating new collective works for resale or redistribution to servers or lists, } \\
\text { or to reuse any copyrighted component of this work in other works must be obtained from the IEEE." }\end{array}$ \\
\hline Type & article \\
\hline File Information & IPTL13-12.pdf \\
\hline
\end{tabular}

Instructions for use 


\title{
Numerical Verification of Degeneracy in Hexagonal Photonic Crystal Fibers
}

\author{
Masanori Koshiba, Senior Member, IEEE, and Kunimasa Saitoh, Member, IEEE
}

\begin{abstract}
Modal birefringences in photonic crystal fibers with six air holes symmetrically arranged around the core region and with multiple air holes in hexagonal lattice are numerically investigated in detail. It is confirmed from computed results obtained by a full-vector finite element method that both the hexagonal holey fibers are not birefringent, namely, the two fundamental modes are degenerate.
\end{abstract}

Index Terms-Finite element method, full vector model, holey fiber, modal birefringence, photonic crystal fiber.

\section{INTRODUCTION}

$\mathbf{P}$ HOTONIC crystal fibers (PCFs), also called holey fibers (HFs) [1] with multiple air holes periodically arranged around the core possess numerous unusual properties, such as wide single-mode wavelength range [2], bend-loss edge at short wavelength [2], large effective core area at single-mode region [3], anomalous group-velocity dispersion at visible and near-infrared wavelengths [4], and strong wavelength-dependent beam divergence [5]. To accurately model HFs, especially with large air holes, it is crucial to use a full vector model [6]-[9]. In particular, a complete vector model is required to predict sensitive quantities such as dispersion and birefringence. Although birefringence between the two fundamental modes in HFs has been very often observed experimentally [10]-[12], the existence of birefringence in HFs has not been fully investigated theoretically/numerically. In [7], [9], it is suggested that hexagonal PCFs are birefringent, but the numerical data are not shown. More recently, a full vector model has been applied to a fiber with a ring of six air holes symmetrically arranged around the core, guaranteeing that such fibers are not birefringent [13].

So far, a full vector model for HFs is based on a modal decomposion approach using sinusoidal functions [plane-wave expansion (PWE) method] [6], [7], Hermite-Gaussian functions (localized function method: LFM) [8], [9], or cylindrical functions [multipole method (MM)] [13]. These methods can accurately model HFs. However, PWE is not efficient, as it does not take advantage of the localization of guided modes. LFM and MM, on the other hand, cannot efficiently describe an extended hexagonal lattice structure [9], and in [13], only the six-hole fiber is treated. Therefore, another method based on space-domain-division-type technique, such as finite element method

Manuscript received June 5, 2001. This work was supported in part by a Grant-in-Aid for Scientific Research from the Ministry of Education, Science, and Culture, Japan.

The authors are with the Division of Electronics and Information Engineering, Graduate School of Engineering, Hokkaido University, 060-8628 Sapporo, Japan.

Publisher Item Identifier S 1041-1135(01)09976-1.

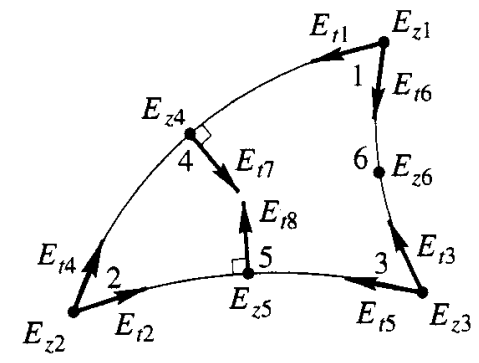

Fig. 1. Curvilinear hybrid edge/nodal element.

(FEM) with locally variable mesh is also useful for design and modeling of HFs and for double check of modal degeneracies of HFs treated in [13].

In this letter, a full vector FEM is applied to HFs, and the modal birefringence is numerically investigated in detail, not only for a fiber with a ring of six air holes, but for a fiber with multiple air holes in hexagonal lattice. As a result, we conclude that both the hexagonal HFs are not birefringent, namely, the two fundamental modes are degenerate.

\section{COMPuted Results AND Discussion}

When applying a full vector FEM to HFs, a curvilinear hybrid edge/nodal element, as shown in Fig. 1, is very useful for avoiding spurious solutions and for accurately modeling curved boundaries of circular air holes. For the axial electric field, $E_{z}$, a nodal element with six variables $E_{z 1}$ to $E_{z 6}$ is employed, while for the transverse electric fields $E_{x}$ and $E_{y}$ an edge element with eight variables $E_{t 1}$ to $E_{t 8}$ is employed, resulting in significantly fast convergence of solutions [14]. ${ }^{1}$

First, we consider a fiber with a ring of six air holes symmetrically arranged around the core as shown in Fig. 2(a), where the hole diameter $d=5 \mu \mathrm{m}$, the hole pitch $\Lambda=6.75 \mu \mathrm{m}$, and the background index $n=1.45$ [13]. Because of the symmetry nature of the system, only one-quarter of the fiber cross section is divided into curvilinear hybrid elements, and the computational window size $R=40.5 \mu \mathrm{m}$.

Fig. 3 shows the convergence behavior of the effective indexes $n_{\text {eff }}^{x}$ and $n_{\text {eff }}^{y}$ for the fundamental $\mathrm{HE}_{11}^{x}$ and $\mathrm{HE}_{11}^{y}$ modes, which are, respectively, approximately uniformly polarized along horizontal $(x)$ and vertical $(y)$ directions, where the operating wavelength $\lambda=1.55 \mu \mathrm{m}$ and "degrees of freedom" stand for the sum of the edge $\left(E_{t}\right)$ and the nodal $\left(E_{z}\right)$ variables used in the whole analysis region. In Fig. 3,

${ }^{1}$ In Table II, LT/QT-2 vector-based shape functions should be corrected as follows: $4|J|_{4}\left|\nabla_{t} L_{1}\right|_{4} L_{1}\left(L_{2} \nabla_{t} L_{3}-L_{3} \nabla_{t} L_{2}\right)$ for $\phi_{t 7}$ and $4|J|_{5}\left|\nabla_{t} L_{2}\right|_{5} L_{2}\left(L_{3} \nabla_{t} L_{1}-L_{1} \nabla_{t} L_{3}\right)$ for $\phi_{t 8}$ 


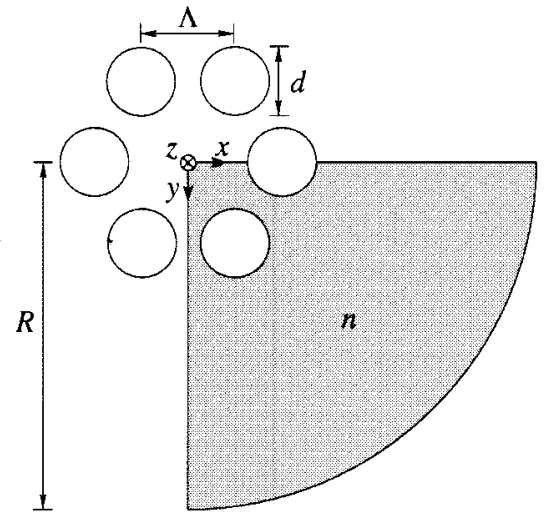

(a)

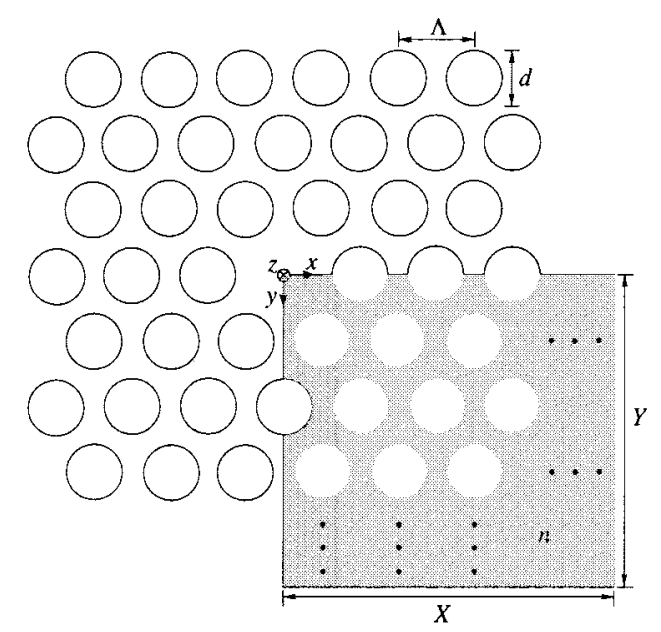

(b)

Fig. 2. PCFs with (a) a ring of six air holes and (b) multiple air holes in hexagonal lattice.

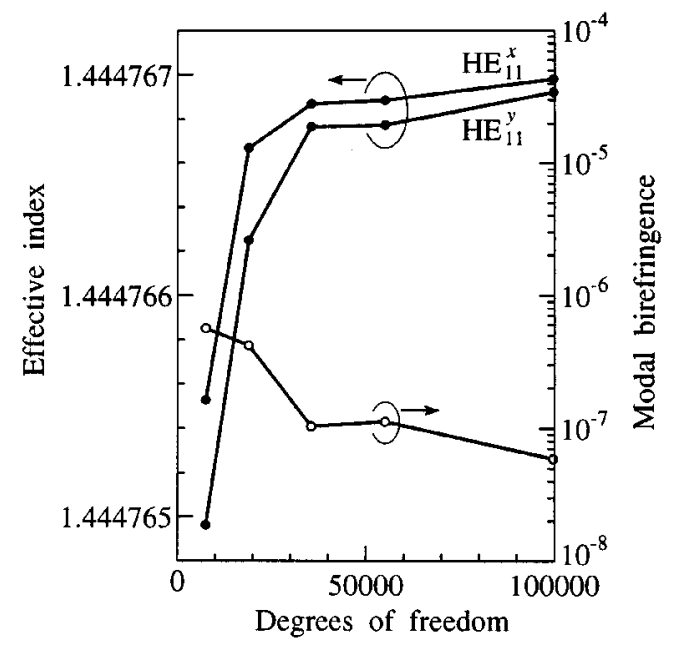

Fig. 3. Convergence of effective indexes and modal birefringence for the $\mathrm{HE}_{11}^{x}$ and the $\mathrm{HE}_{11}^{y}$ modes in a six-hole fiber.

the modal birefringence $n_{\mathrm{eff}}^{x}-n_{\mathrm{eff}}^{y}$ is also plotted. We find degeneracy in the $\mathrm{HE}_{11}$ states to the order of $10^{-8}$ with degrees of freedom of 99687, corresponding to 14198 elements. This level is almost the same as that reported in [13]. Fig. 4 shows the transverse electric field vector distributions. We can see that each fundamental mode is essentially a linearly polarized field.

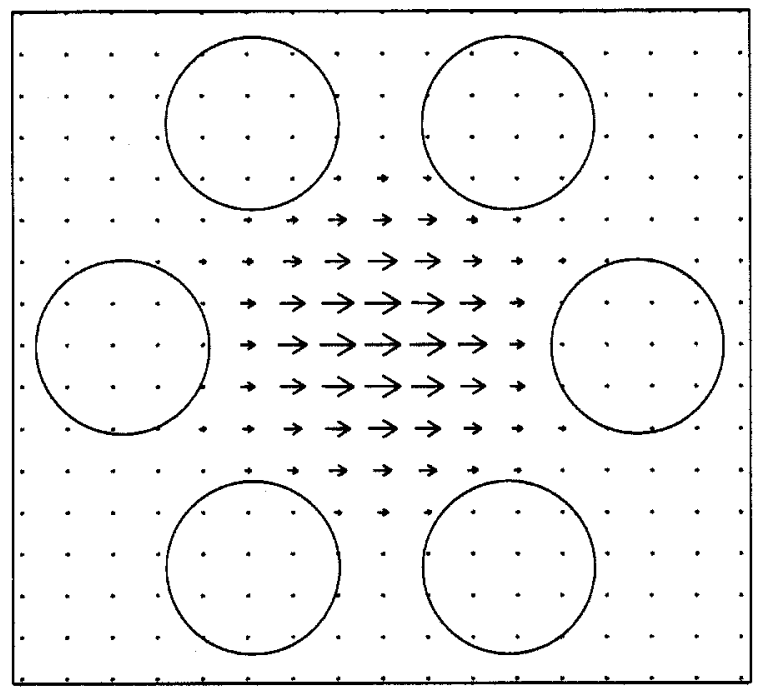

(a)

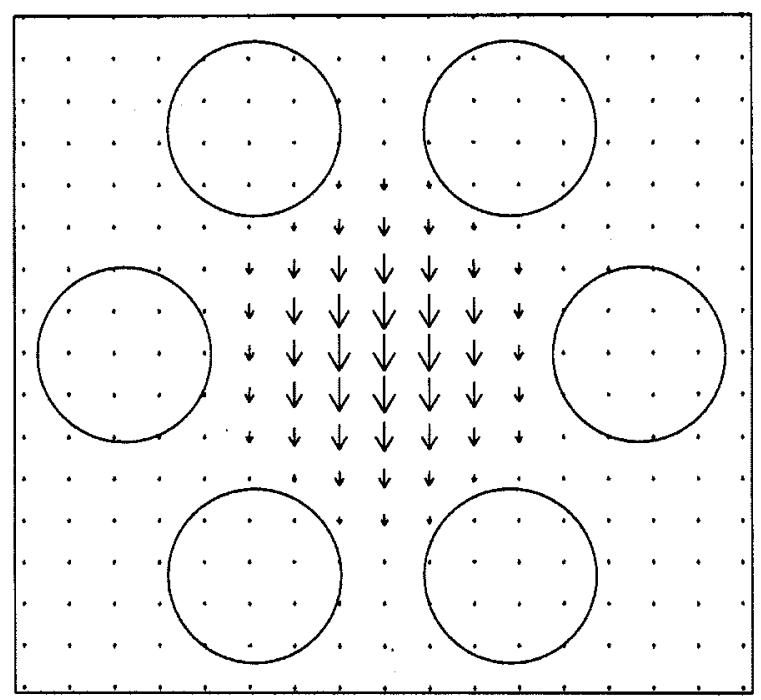

(b)

Fig. 4. Transverse electric field vectors for (a) the $\mathrm{HE}_{11}^{x}$ and (b) the $\mathrm{HE}_{11}^{y}$ modes in a six-hole fiber.

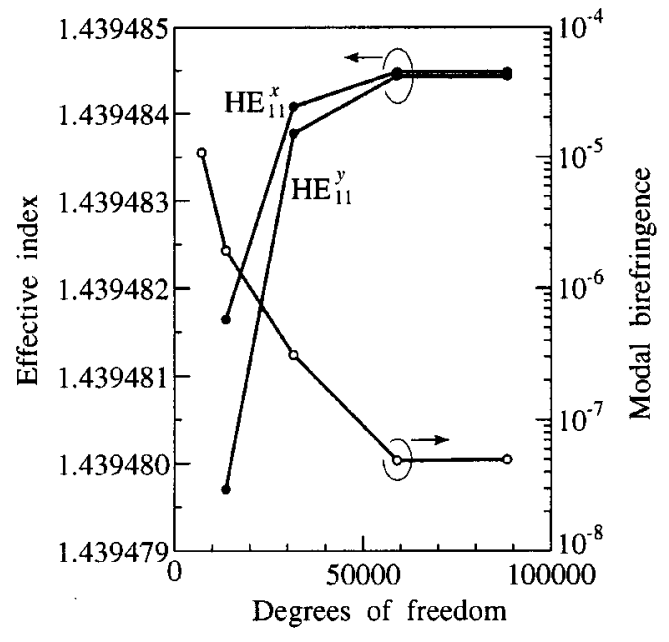

Fig. 5. Convergence of effective indexes and modal birefringence for the $\mathrm{HE}_{11}^{x}$ and the $\mathrm{HE}_{11}^{y}$ modes in a hexagonal-lattice-cladding fiber. 


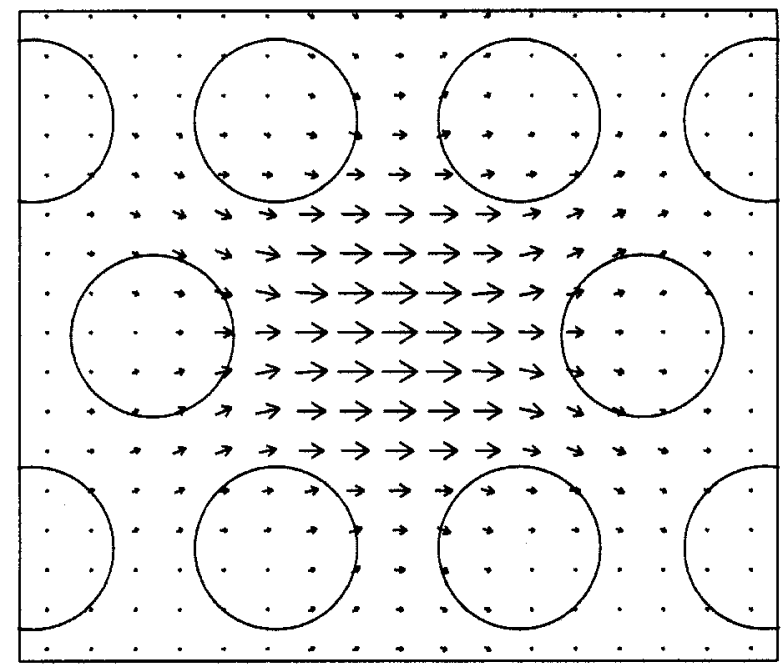

(a)

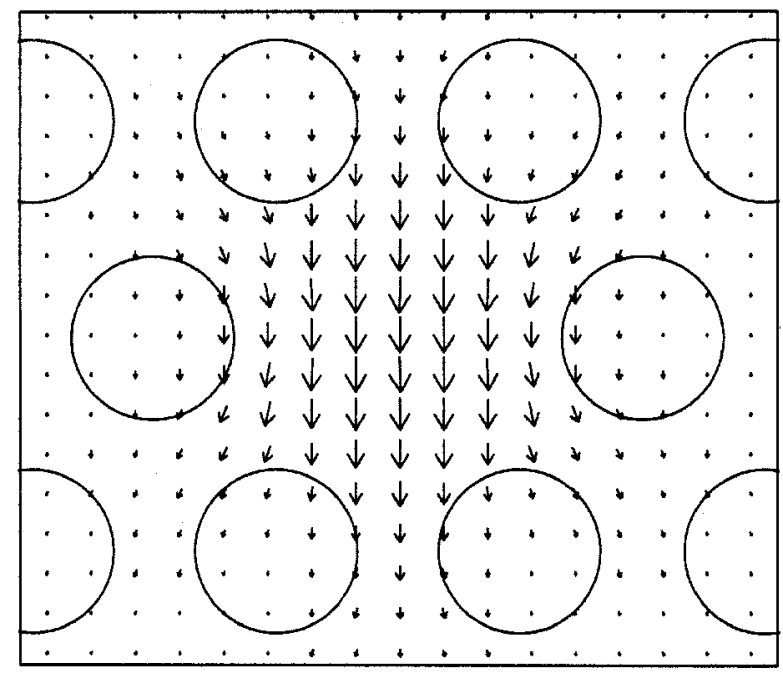

(b)

Fig. 6. Transverse electric field vectors for (a) the $\mathrm{HE}_{11}^{x}$ and (b) the $\mathrm{HE}_{11}^{y}$ modes in a hexagonal-lattice-cladding fiber.

Next, we consider a fiber with multiple air holes in hexagonal lattice as shown in Fig. 1(b), where the hole diameter $d=1.51$ $\mu \mathrm{m}$, the hole pitch $\Lambda=2.26 \mu \mathrm{m}$, the background index $n=$ 1.45 , and the operating wavelength $\lambda=0.8 \mu \mathrm{m}$ [15]. Because of the symmetry nature of the system, only one-quarter of the fiber cross section is divided into curvilinear hybrid elements, and the computational window size $X=10.17 \mu \mathrm{m}$ and $Y=$ $9.13 \mu \mathrm{m}$.

Fig. 5 shows the convergence behavior of the refractive indexes $n_{\mathrm{eff}}^{x}$ and $n_{\mathrm{eff}}^{y}$ and the modal birefringence $n_{\mathrm{eff}}^{x}-n_{\mathrm{eff}}^{y}$. Also in this fiber, we again find degeneracy in the $\mathrm{HE}_{11}$ states to the level of $4.9 \times 10^{-8}$ with degrees of freedom of 88345 , corresponding to 12576 elements. Fig. 6 shows the transverse electric field vector distributions.

Noting that the hexagonal PCF has six-fold rotational symmetry and applying $2 \pi / m$ rotation ( $m=1$ to 6 ) to the $\mathrm{HE}_{11}^{x}$ (or $\mathrm{HE}_{11}^{y}$ ) field, we obtain the rotated field which must be a lin- early polarized mode with the original effective index $n_{\text {eff }}^{x}$ (or $n_{\text {eff }}^{y}$ ). This rotated field can also be expressed as a superposition of the two orthogonal $\mathrm{HE}_{11}^{x}$ and $\mathrm{HE}_{11}^{y}$ states. If the fiber is not degenerate, namely $n_{\mathrm{eff}}^{x} \neq n_{\mathrm{eff}}^{y}$, we cannot obtain the above rotated field with linear polarization and effective index $n_{\mathrm{eff}}^{x}$ (or $n_{\text {eff }}^{y}$ ). As a result, fibers with six-fold rotational symmetry are not birefringent.

\section{CONCLUSION}

A full vector FEM with curvilinear hybrid edge/nodal elements was effectively applied not only to a fiber with six air holes, but to a fiber with multiple air holes in hexagonal lattice. It was confirmed from numerical results that both the hexagonal holey fibers are not birefringent, namely, the two fundamental modes are degenerate.

\section{REFERENCES}

[1] J. C. Knight, T. A. Birks, P. St. J. Russell, and D. M. Atkin, "All-silica single-mode optical fiber with photonic crystal cladding," Opt. Lett., vol. 21, pp. 1547-1549, Oct. 1996.

[2] T. A. Birks, J. C. Knight, and P. St. J. Russell, "Endlessy single-mode photonic crystal fiber," Opt. Lett., vol. 22, pp. 961-963, July 1997.

[3] J. C. Knight, T. A. Birks, R. F. Cregan, P. St. J. Russell, and J.-P. de Sandro, "Large mode area photonic crystal fiber," Electron. Lett., vol. 34, pp. 1347-1348, June 1998.

[4] M. J. Gander, R. McBride, J. D. C. Jones, D. Mogilevtsev, T. A. Birks, J. C. Knight, and P. St. J. Russell, "Experimantal measurement of group velocity in photonic crystal fiber," Electron. Lett., vol. 35, pp. 63-64, Jan. 1999.

[5] M. J. Gander, R. McBride, J. D. C. Jones, T. A. Birks, J. C. Knight, P. St. J. Russell, P. M. Blanchard, J. G. Burnett, and A. H. Greenaway, "Measurement of the wavelength dependence of beam divergence for photonic crystal fiber," Opt. Lett., vol. 24, pp. 1017-1019, Aug. 1999.

[6] A. Ferrando, E. Silvestre, J. J. Miret, P. Andrés, and M. V. Andrés, "Fullvector analysis of a realistic photonic crystal fiber," Opt. Lett., vol. 24, pp. 276-278, Mar. 1999.

[7] -, "Vector description of higher-order modes in photonic crystal fibers," J. Opt. Soc. Amer. A, vol. 17, pp. 1333-1340, July 2000.

[8] D. Mogilevtsev, T. A. Birks, and P. St. J. Russell, "Localized function method for modeling defect modes in 2-D photonic crystals," J. Lightwave Technol., vol. 17, pp. 2078-2081, Nov. 1999.

[9] T. M. Monro, D. J. Richardson, N. G. R. Broderick, and P. J. Bennett, "Modeling large air fraction holey optical fibers," J. Lightwave Technol., vol. 18, pp. 50-56, Jan. 2000.

[10] J. K. Ranka, R. S. Windeler, and A. J. Stentz, "Visible continuum generation in air-silica microstructure optical fibers with anomalous dispersion at $800 \mathrm{~nm}$," Opt. Lett., vol. 25, pp. 25-27, Jan. 2000.

[11] J. C. Knight, J. Arriaga, T. A. Birks, A. Ortigosa-Blanch, W. J. Wadsworth, and P. St. J. Russell, "Anomalous dispersion in photonic crystal fiber," IEEE Photon. Technol. Lett., vol. 12, pp. 807-809, July 2000.

[12] B. J. Eggleton, P. S. Westbrook, C. A. White, C. Kerbage, R. S. Windeler, and G. L. Burdge, "Cladding-mode resonances in air-silica microstructure optical fibers," J. Lightwave Technol., vol. 18, pp. 1084-1100, Aug. 2000.

[13] M. J. Steel, T. P. White, C. Martijn de Sterke, R. C. McPhedran, and L. C. Botten, "Symmetry and degeneracy in microstructured optical fibers," Opt. Lett., vol. 26, pp. 488-490, Apr. 2001.

[14] M. Koshiba and Y. Tsuji, "Curvilinear hybrid edge/nodal elements with triangular shape for guided-wave problems," J. Lightwave Technol., vol. 18, pp. 737-743, May 2000.

[15] H. Kubota, K. Suzuki, S. Kawashima, M. Nakazawa, M. Tanaka, and M. Fujita, "Low-loss, $2 \mathrm{~km}$-long photonic crystal fiber with zero GVD in the near IR suitable for picosecond pulse propagation at the $800 \mathrm{~nm}$ band," in Proc. Conf. Laser Electrooptics, Baltimore, MD, 2001, Postdeadline paper CPD3-1. 